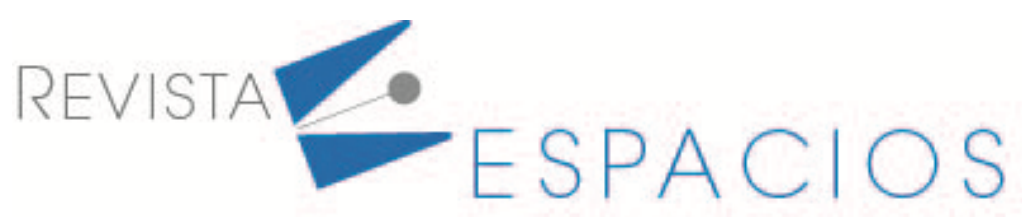

\title{
Relación entre ansiedad escolar y perfeccionismo en una muestra de alumnado ecuatoriano
}

\section{Relationship between school anxiety and perfectionism in a sample of Ecuadorian students}

\author{
ÁLVAREZ TERUEL, José D. ${ }^{1}$ \\ JIMENEZ AYALA, Carlos E. ${ }^{2}$ \\ CARGUA GARCÍA, Nancy ${ }^{3}$ \\ APARICIO-FLORES, María P. ${ }^{4}$ \\ FERNANDEZ-SOGORB, Aitana ${ }^{5}$ \\ GARCIA-FERNANDEZ, José M. ${ }^{6}$
}

\section{Resumen}

La ansiedad escolar es uno de los trastornos emocionales con mayor prevalencia mundial en todas las edades. El presente estudio tuvo como objetivo examinar la relación de la ansiedad escolar con el perfeccionismo, considerado como un factor de vulnerabilidad psicológico que afecta directamente al rendimiento académico, en una muestra de 1588 estudiantes ecuatorianos de 12 a 18 años $(M=14.82 ; D . E$. $=1.86)$. Se utilizaron el Inventario de Ansiedad Escolar (IAES) y la Child and Adolescent Perfectionism Scale (CAPS). El análisis de los datos reveló que el alumnado con alta ansiedad escolar mostró niveles significativamente más altos de perfeccionismo y que altos niveles de predicen altos niveles de ansiedad escolar.

Palabras clave: ansiedad escolar; perfeccionismo; inventario de ansiedad escolar (IAES); escolares ecuatorianos.

\footnotetext{
Abstract

School anxiety is one of the emotional disorders with the highest worldwide prevalence at all ages. The present study aimed to examine the relationship of school anxiety with perfectionism, which is considered as a factor of psychological vulnerability that directly affects academic performance, in a sample of 1588 Ecuadorian students aged 12 to 18 years $(M=14.82 ;$ S.D. $=1.86)$. The School Anxiety Inventory (IAES) and the Child and Adolescent Perfectionism Scale (CAPS) were used. Data analysis revealed that students with high school anxiety showed significantly higher levels of perfectionism and that high levels of perfectionism predict high levels of school anxiety.

key words: school anxiety; perfectionism; school anxiety inventory (IAES); Ecuadorian schoolchildren.

${ }^{1}$ Profesor Contratado Doctor. Departamento de Psicología Evolutiva y Didáctica. Universidad de Alicante. josedaniel.alvarez@ua.es ${ }^{2}$ Profesor. Faculty of Philosophy, Literature and Educational Sciences, Central University of Ecuador, Ecuador. cejimenez@uce.edu.ec ${ }^{3}$ Profesora. Faculty of Philosophy, Literature and Educational Sciences, Central University of Ecuador, Ecuador.ncargua@uce.edu.ec

${ }^{4}$ Profesora Asociada (LOU). Departamento de Didáctica General y Didácticas Específicas. Universidad de Alicante. pilar.aparicio@ua.es

${ }^{5}$ Investigadora Predoctoral. Departamento de Psicología Evolutiva y Didáctica. Universidad de Alicante. aitana.fernandez@ua.es

${ }^{6}$ Profesor Titular de Universidad. Departamento de Psicología Evolutiva y Didáctica. Universidad de Alicante. josemagf@ua.es
} 


\section{Introducción}

Dos aspectos fundamentales en el desarrollo de la niñez y la adolescencia centran la atención de este estudio: la ansiedad escolar y el perfeccionismo.

La ansiedad escolar es uno de los problemas emocionales que genera un gran interés a familias, docentes, y profesionales del ámbito de la psicología y la medicina, ya que se considera uno de los trastornos emocionales más prevalente a nivel mundial, tanto en la infancia, la adolescencia, como en la edad adulta (Costello, Egger y Angold, 2004; Demyttenaere et al., 2004; In-Albon y Scheneider, 2007; Mardomingo, 2005; Mazzone et al., 2007; Ollendick y Seligman, 2005; Vasa y Pine, 2006).

En Ecuador, ámbito del estudio, se observa como cada día más estudiantes presentan problemas de ansiedad que no son atendidos adecuadamente, por lo que esta cuestión se convierte en un centro de interés importante para la investigación.

La presencia de sintomatología ansiosa en la infancia y en la adolescencia predice, en muchos casos, el desarrollo posterior y la cronicidad de un trastorno de ansiedad (McLoone, Hudson y Rapee, 2006; Kessler et al., 2005). De este modo, el origen de los trastornos de ansiedad se sitúa en la niñez, y estos aumentan considerablemente en torno a los once años, convirtiéndose en uno de los problemas emocionales con mayor repercusión en el desarrollo del ser humano a edades tempranas. Cada niño y adolescente muestra distintos síntomas ansiosos y con diversa intensidad, variando en su persistencia y en el periodo en que se presentan, y obedeciendo a tres sistemas de respuestas: motora; psicofisiológicas y cognitivas. Pero no todos experimentan el mismo patrón o intensidad de la sintomatología, variando las reacciones en función de su duración, persistencia y fluctuación temporal.

El alumnado se enfrenta diariamente a situaciones de gran presión como la adaptación a nuevos compañeros; a nuevos profesores; a evaluaciones exigentes; a la diversidad o el aprendizaje de contenidos. Estos aspectos contribuyen al aumento de los miedos y ansiedad relacionados con la escuela en la niñez (Rosário, Mourão, Núñez, González-Pienda y Solano, 2008; Wren y Benson, 2004). Entre las situaciones escolares que provocan mayores niveles de ansiedad, se encuentran: las evaluaciones (académicas y sociales); las situaciones de fracaso y castigo escolar; o las situaciones de agresión (García-Fernández, Inglés, Martínez-Monteagudo, Marzo y Estévez, 2011). Por eso resulta importante al evaluar la respuesta de ansiedad escolar, que se tengan en cuenta no sólo las distintas manifestaciones que componen esta reacción compleja, sino también las múltiples variables psicoeducativas que inciden en el rendimiento académico.

La disminución en el rendimiento en la escuela tiene consecuencias negativas tanto en las calificaciones como en la autoestima de los estudiantes. Cogniciones específicas pueden desarrollar la percepción de situaciones como algo hostil y amenazador, y pueden provocar que el estudiante corra el riesgo de desarrollar o mantener trastornos ansiosos. Asimismo, si la ansiedad es intensa, el estudiante muestra excesiva sensibilidad y temores, incluyendo temor a la escuela, vergüenza, timidez, sensaciones importantes de inseguridad y síntomas somáticos. El alumnado intensamente ansioso padece de una extraordinaria tendencia a preocuparse excesivamente por su competencia cognoscitiva y rendimiento escolar, exteriorizando intranquilidad, trastornos del sueño, dolores de estómago o alteraciones conductuales, entre otros. Sienten temor de no gustarle a los demás, sufren la sensación de que sus compañeros se ríen de ellos y se muestran aprensivos frente a actividades que otros alumnos realizan con entusiasmo.

Las diversas investigaciones demuestran que los niños y adolescentes que manifiestan fobia escolar tienden a presentar un rendimiento académico más bajo (King y Bernstein, 2001), dolores de cabeza crónicos (Fujita et al., 2009), experiencias de bullying (Martínez-Monteagudo, Inglés, Trianes y García-Fernández, 2011) sentimientos 
negativos acerca de su autoreferencia (Muris, Merckelbach, de Jong y Ollendick, 2002), problemas de sueño (Gregory y Eley, 2005), y otros trastornos de ansiedad comórbidos como la ansiedad por separación (Orgilés, Espada, García-Fernández y Méndez, 2009), desesperanza, tristeza, ambivalencia e hiperactividad, mayores índices de neuroticismo y menores de extraversión, así como una autoestima y autoconcepto negativos (HaraldFreudenthaler et al., 2008; Putwain y Daniels, 2010).

\subsection{Objetivos de la investigación}

El objetivo general de la investigación será confirmar la estructura factorial del Inventario de Ansiedad Escolar (IAES) en población ecuatoriana y establecer su relación con el perfeccionismo en una muestra de estudiantes ecuatorianos de 12 a 18 años.

Concretamente nuestro trabajo se centrará en:

1. Analizar las diferencias en perfeccionismo en una muestra de adolescentes ecuatorianos con alta y baja ansiedad escolar.

2. Determinar la influencia del perfeccionismo sobre la ansiedad escolar en una muestra de adolescentes ecuatorianos.

Para ello nos planteamos dos hipótesis de trabajo:

1. Se espera que los estudiantes con alta ansiedad escolar, en comparación con sus compañeros con baja ansiedad escolar, presenten puntuaciones significativamente más altas en las dimensiones de perfeccionismo.

2. Se espera que a medida que aumentan las puntuaciones en perfeccionismo aumente la probabilidad de presentar alta ansiedad escolar.

\section{Metodología}

\subsection{Participantes}

La muestra de sujetos estuvo formada por 1725 estudiantes con edades comprendidas entre los 12 y 18 años, matriculados en los cursos comprendidos desde octavo hasta tercero de bachillerato en ocho centros, de los que 137 fueron eliminados por errores en las respuestas o por no tener el consentimiento de las familias, quedando compuesta la muestra por 1588 sujetos $(M=14.82$; D.E. $=1.86)$. La selección de los centros participantes se llevó a cabo a través de un muestreo por conglomerados en la ciudad de Quito (véase tabla $N^{\circ} 1$ ).

Tabla 1

Número (porcentaje) de participantes de la muestra

total clasificados en función del sexo y el curso

\begin{tabular}{|c|c|c|c|c|c|c|c|}
\hline & Octavo & Noveno & Décimo & 1 $\mathrm{BACH}$ & 20 $\mathrm{BACH}$ & 3 $\mathrm{BACH}$ & Total \\
\hline \multirow{3}{*}{ Varones } & 152 & 162 & 167 & 150 & 145 & 191 & 967 \\
\cline { 2 - 8 } & $9.6 \%$ & $10.2 \%$ & $10.5 \%$ & $9.4 \%$ & $9.1 \%$ & $12.0 \%$ & $60.9 \%$ \\
\hline \multirow{3}{*}{ Mujeres } & 116 & 144 & 123 & 60 & 80 & 98 & 621 \\
\cline { 2 - 8 } & $7.3 \%$ & $9.1 \%$ & $7.7 \%$ & $3.8 \%$ & $5.0 \%$ & $6.2 \%$ & $39.1 \%$ \\
\hline \multirow{3}{*}{ Total } & 268 & 306 & 290 & 210 & 225 & 289 & 1588 \\
\cline { 2 - 9 } & $16.9 \%$ & $19.3 \%$ & $18.3 \%$ & $13.2 \%$ & $14.2 \%$ & $18.2 \%$ & $100.0 \%$ \\
\hline
\end{tabular}

Fuente: Elaboración propia 


\subsection{Instrumentos}

Inventario de Ansiedad Escolar (IAES), García-Fernández, Inglés, Martínez-Monteagudo, Marzo y Estévez (2011).

El IAES es un instrumento que evalúa el nivel de ansiedad en adolescentes de edades comprendidas entre los 12 y los 18 años. Su estructura está conformada por 25 ítems referidos a situaciones escolares, ubicadas en el eje horizontal de una tabla de doble entrada y 19 respuestas ( 9 de tipo cognitivas, 5 de tipo conductuales y 5 de tipo psicofisiológicas) ubicadas en el eje vertical de esta misma tabla. Para contestar el sujeto debe valorar la respuesta en función de la situación a partir de una escala de 5 puntos (0: nunca, 4: siempre).

Los análisis psicométricos del IAES indicaron la existencia de 4 factores correlacionados (Ansiedad ante el fracaso y castigo escolar, Ansiedad ante la evaluación social, Ansiedad ante la agresión y Ansiedad ante la evaluación escolar) y tres factores de respuesta independientes (Ansiedad cognitiva, Ansiedad psicofisiológica y Ansiedad conductual). El análisis de fiabilidad de los tres sistemas de respuesta reveló coeficientes de consistencia interna adecuados; entre 0.97 (Ansiedad cognitiva y Ansiedad conductual) y 0.98 (Ansiedad psicofisiológica), y los coeficientes de fiabilidad test-retest, para un intervalo de 4 semanas, variaron de 0.79 (Ansiedad psicofisiológica) a 0.82 (Ansiedad conductual).

Child and Adolescent Perfectionism Scale (CAPS); Flett et al., (2000).

La CAPS es una medida de autoinforme compuesta por 22 ítems que evalúa dos dimensiones del perfeccionismo, Perfeccionismo Auto-Orientado (PAO) y Perfeccionismo Socialmente Prescrito (PSP), en niñas, niños y adolescentes. Los ítems se responden a través de una escala tipo Líkert de 5 puntos. La validación original de la escala obtuvo adecuados índices de fiabilidad ( $\alpha=.81$, para PAO y $\alpha=.85$ para PSP).

\subsection{Procedimiento}

Después de establecer los objetivos de la investigación, se llevó a cabo una entrevista con directores, jefes de estudios y coordinadores de los equipos de orientación y apoyo de los centros participantes con el propósito de exponer los objetivos de la investigación, describir los instrumentos de evaluación, solicitar permiso y promover su colaboración.

Posteriormente, se envió una carta informativa a las familias del alumnado para explicarles el estudio y solicitar el consentimiento informado por escrito, autorizando así a sus hijos a participar en la investigación. Tras el proceso de selección de la muestra de participantes y la obtención del consentimiento firmado, se procedió a la administración de las pruebas.

Los instrumentos se explicaron previamente al alumnado participante, se administraron de manera voluntaria y colectiva en el aula, con la ayuda del profesorado tutor de cada uno de los cursos y la presencia del investigador para posible aclaración de dudas y verificación de la fiabilidad de las respuestas.

Primeramente, el alumnado llenó los datos personales: sexo, edad, curso escolar y centro. En segundo lugar, se explicaron en voz alta las instrucciones de los cuestionarios con el fin de resolver posibles dudas y enfatizar en la importancia de responder a todas y cada una de las preguntas. Seguidamente, se pasó a la cumplimentación de las pruebas.

\subsection{Diseño y análisis de datos}

A continuación, se especifican los análisis estadísticos utilizados para poner a prueba las hipótesis planteadas en el estudio: 
- Para analizar las diferencias entre estudiantes con alta y baja ansiedad escolar en las puntuaciones de los cuestionarios sobre perfeccionismo se utilizó la prueba $t$ de diferencia de medias para medidas independientes. Además de atender a la significatividad de las diferencias de medias resulta fundamental conocer el tamaño de estas diferencias puesto que, en el caso de que se trabaje con un elevado tamaño muestral, se pueden detectar diferencias significativas erróneamente. Para analizar el tamaño del efecto se halló el índice d propuesto por Cohen (1988).

- Para analizar la relación entre la ansiedad escolar y el perfeccionismo se utilizó la regresión logística, siguiendo el procedimiento por pasos hacia delante basado en el estadístico de Wald, para ello se dicotomizaron las variables de ansiedad escolar considerando bajas puntuaciones las que estaban por debajo del percentil 25 y altas las que estaban por encima del percentil 75 . El que se opte por el uso de la regresión logística frente a la lineal se debe, entre otros motivos, a que dicotomizar las variables como criterio (Alderete, 2006) y evita algunos de los problemas que pueden provocar la aplicación de los modelos lineales cuando las variables dependientes son binarias.

- Para los análisis se utilizaron los paquetes estadísticos SPSS versión 20 y AMOS 20.

\section{Resultados}

\subsection{Diferencias en perfeccionismo en estudiantes con altas y bajas puntuaciones en Ansiedad Cognitiva}

La Tabla 2 y la Figura 1 ofrecen las medias y diferencias estadísticamente significativas en perfeccionismo halladas en estudiantes con altas y bajas puntuaciones en ansiedad escolar en el Factor IV (Ansiedad Cognitiva) del IAES.

Las diferencias halladas resultaron significativas en PSP $=$ Perfeccionismo Socialmente Prescrito y PAO = Perfeccionismo Auto-orientado, con una magnitud baja para ambas dimensiones ( $d=.48$ y .33 respectivamente). El alumnado con altas puntuaciones en Ansiedad Cognitiva puntuó significativamente más alto que sus iguales con bajas puntuaciones.

Tabla 2

Diferencias en perfeccionismo en estudiantes con altas y bajas puntuaciones en Ansiedad Cognitiva

\begin{tabular}{|l|c|c|c|r|r|r|r|r|r|r|}
\hline \multirow{2}{*}{ Variable } & \multicolumn{2}{|c|}{$\begin{array}{c}\text { Prueba } \\
\text { Levene }\end{array}$} & \multicolumn{2}{c|}{$\begin{array}{c}\text { Altas } \\
\text { Puntuaciones }\end{array}$} & \multicolumn{2}{c|}{$\begin{array}{c}\text { Bajas } \\
\text { puntuaciones }\end{array}$} & \multicolumn{4}{c|}{$\begin{array}{c}\text { Significación estadística y } \\
\text { magnitud diferencias }\end{array}$} \\
\hline & $F$ & $P$ & $M$ & $D E$ & $M$ & $D E$ & $t$ & $g . l$. & \multicolumn{1}{c|}{$p$} & $d$ \\
\hline PSP & 6.28 & .012 & 34.44 & 6.28 & 31.25 & 7.18 & -6.66 & 747.46 & $<.001$ & .48 \\
\hline PAO & 1.44 & .229 & 40.54 & 6.37 & 38.39 & 6.67 & -4.67 & 804 & $<.001$ & .33 \\
\hline
\end{tabular}

Nota: PSP = Perfeccionismo Socialmente Prescrito, PAO = Perfeccionismo Auto-orientado

Fuente: Elaboración propia. 
Figura 1

Diagrama de barras de las diferencias en perfeccionismo en estudiantes altas y bajas puntuaciones en el Factor I del IAES,

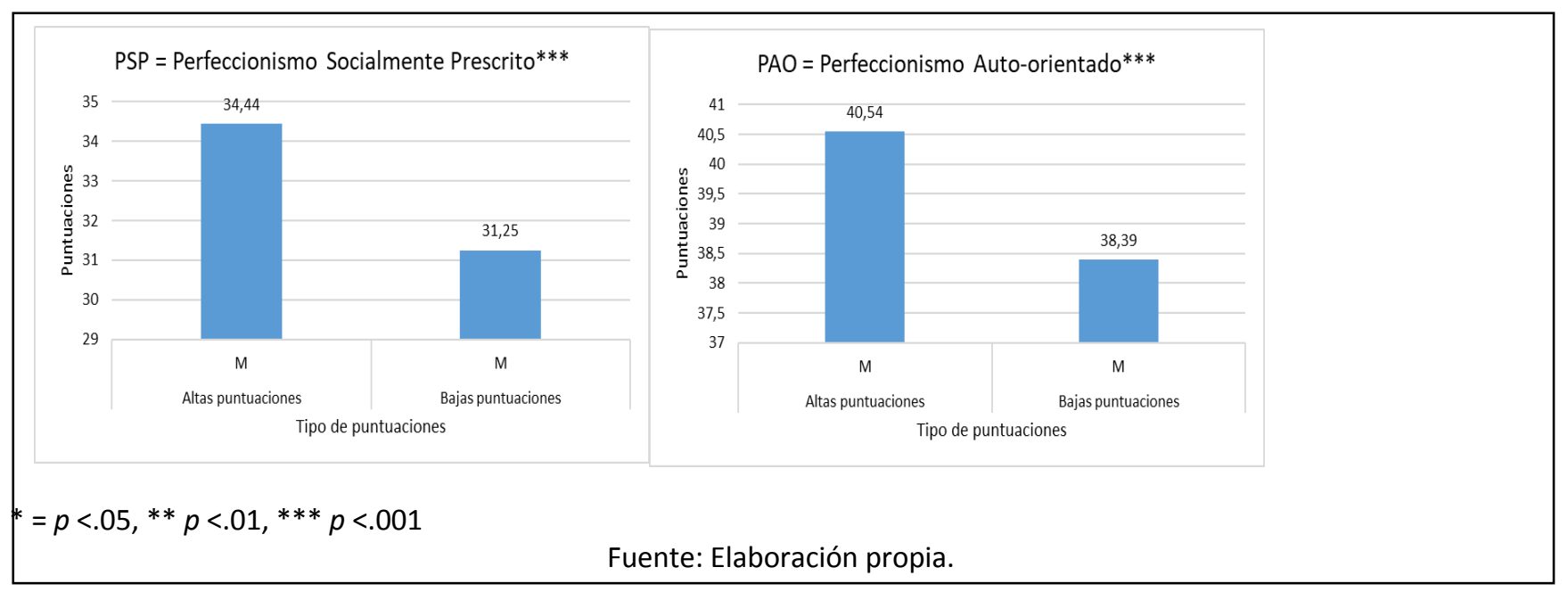

\subsection{Regresión logística binaria para la probabilidad de presentar alta puntuación en Ansiedad Cognitiva en función de las variables de perfeccionismo}

Los modelos resultantes (véase Tabla 3) permiten hacer una estimación correcta del $59.2 \%$ de los casos ( $\chi 2=$ $21.57 ; p=<.05)$ para la variable PAO y de un $60.3 \%$ de los casos $(\chi 2=43.82 ; p=<.05)$ correspondiente a la variable PSP. El valor de ajuste de los modelos (R2 Nagelkerke) se situó en el .04 para la variable PAO y en .07 para la variable PSP.

Los valores de la odd ratio $(O R)$ fueron superiores a 1 indicando, por tanto, que a medida que aumenta la puntuación en las variables de perfeccionismo aumenta la posibilidad de presentar altas puntuaciones en Ansiedad Cognitiva, obteniendo valores para la variable PAO de 1.05 y de 1.07 para PSP.

Tabla 3

Regresión logística binaria para la probabilidad de presentar alta puntuación en Ansiedad Cognitiva en función de las variables de perfeccionismo.

\begin{tabular}{|l|l|c|c|c|c|c|c|c|c|}
\hline Variable & & $\chi 2$ & $\mathrm{R} 2$ & $\mathrm{~B}$ & $\mathrm{E} . \mathrm{T}$. & Wald & $p$ & OR & I.C. 95\% \\
\hline PAO & $\begin{array}{l}\text { Clasificados } \\
\text { correctamente: } \\
59.2 \%\end{array}$ & 21.57 & .04 & .05 & .0 & 20.75 & $<.001$ & 1.05 & $1.02-1.07$ \\
\hline & Constante & 43.82 & .07 & .07 & .01 & 40.90 & $<.001$ & 1.07 & $1.05-1.09$ \\
\hline PSP & $\begin{array}{l}\text { Clasificados } \\
\text { correctamente: } \\
60.3 \%\end{array}$ & & & & & & $<.001$ & & \\
\hline & Constante & & & -2.16 & .36 & 34.64 & $<.001$ & .11 & \\
\hline
\end{tabular}

Nota: $\mathrm{PAO}=$ Perfeccionismo Auto-orientado, PSP = Perfeccionismo Socialmente Prescrito, $\chi 2=$ Chi cuadrado; $\mathrm{R} 2$ = Cuadrado de Nagelkerke; $\mathrm{B}=$ Coeficiente de regresión; E.T.= Error estándar; Wald = Prueba de Wald; $p=$ Probabilidad; $O R=$ Odd ratio; I.C. = Intervalo de confianza al 95\%.

Fuente: Elaboración propia. 


\subsection{Modelo de regresión logística binaria para la probabilidad de presentar alta puntuación en Ansiedad Cognitiva en función de los factores de perfeccionismo incluyendo todas las variables}

La Tabla 4 presenta el modelo de regresión logística binaria para Ansiedad Cognitiva resultante tras incluir las variables de perfeccionismo, en donde los resultados muestran que la proporción de casos clasificados correctamente es del 60.3\% ( $\chi 2=43.82 ; p=<.05)$. Los componentes del modelo expresados por la OR permiten pronosticar una alta ansiedad escolar en base a Ansiedad Cognitiva, siendo 1.07 veces mayor la probabilidad de manifestar ansiedad escolar por cada punto que se incrementa la puntuación en la variable PSP.

Tabla 4

Modelo de regresión logística binaria para la probabilidad de presentar alta puntuación en Ansiedad Cognitiva en función de los factores de perfeccionismo incluyendo todas las variables

\begin{tabular}{|c|c|c|c|c|c|c|c|c|c|}
\hline Variable & & $x^{2}$ & $\mathrm{R} 2$ & B & E.T. & Wald & $p$ & OR & I.C. $95 \%$ \\
\hline \multirow[t]{2}{*}{ PSP } & $\begin{array}{c}\text { Clasificados } \\
\text { correctamente: } \\
60.3 \%\end{array}$ & 43.82 & .07 & -2.16 & .36 & 34.64 & \multirow{2}{*}{$\begin{array}{l}<.001 \\
<.001\end{array}$} & .11 & $1.05-1.09$ \\
\hline & Constante & & & 0.7 & .01 & 40.90 & & 1.07 & \\
\hline
\end{tabular}

Nota: PSP = Perfeccionismo Socialmente Prescrito, $\chi_{2}=$ Chi cuadrado; $\mathrm{R}_{2}=$ Cuadrado de Nagelkerke; $\mathrm{B}=$ Coeficiente de regresión; E.T.= Error estándar; Wald = Prueba de Wald; $p=$ Probabilidad; $O R=$ Odd ratio; I.C. = Intervalo de confianza al $95 \%$.

Fuente: Elaboración propia.

\subsection{Diferencias en perfeccionismo en estudiantes con altas y bajas puntuaciones en Ansiedad Conductual}

La Tabla 5 y la Figura 2 ofrecen las medias y diferencias estadísticamente significativas en perfeccionismo halladas en estudiantes con altas y bajas puntuaciones en ansiedad escolar en el Factor V (Ansiedad Conductual) del IAES.

Las diferencias halladas resultaron significativas en PSP y en PAO, con una magnitud baja para ambas dimensiones ( $d=.45$ y .22, respectivamente). El alumnado con altas puntuaciones en Ansiedad Conductual puntuó significativamente más alto que sus iguales con bajas puntuaciones.

Tabla 5

Diferencias en perfeccionismo en estudiantes con altas y bajas puntuaciones en Ansiedad Conductual.

\begin{tabular}{|c|c|c|c|c|c|c|c|c|c|c|}
\hline \multirow[t]{2}{*}{ Variable } & \multicolumn{2}{|c|}{$\begin{array}{l}\text { Prueba } \\
\text { Levene }\end{array}$} & \multicolumn{2}{|c|}{ Altas puntuaciones } & \multicolumn{2}{|c|}{ Bajas puntuaciones } & \multicolumn{4}{|c|}{$\begin{array}{c}\text { Significación estadística y magnitud } \\
\text { diferencias }\end{array}$} \\
\hline & $F$ & $P$ & $M$ & $D E$ & $M$ & $D E$ & $t$ & g.l. & $p$ & $d$ \\
\hline PSP & 4.08 & .044 & 34.22 & 6.32 & 31.26 & 6.98 & -6.12 & 701.98 & $<001$ & .45 \\
\hline PAO & .92 & .336 & 39.85 & 6.43 & 38.43 & 6.68 & -3.01 & 776 & .003 & .22 \\
\hline
\end{tabular}

Nota: PSP = Perfeccionismo Socialmente Prescrito, PAO = Perfeccionismo Auto-orientado

Fuente: Elaboración propia. 
Figura 2

Diagrama de barras de las diferencias en perfeccionismo en estudiantes altas y bajas puntuaciones en Ansiedad Conductual
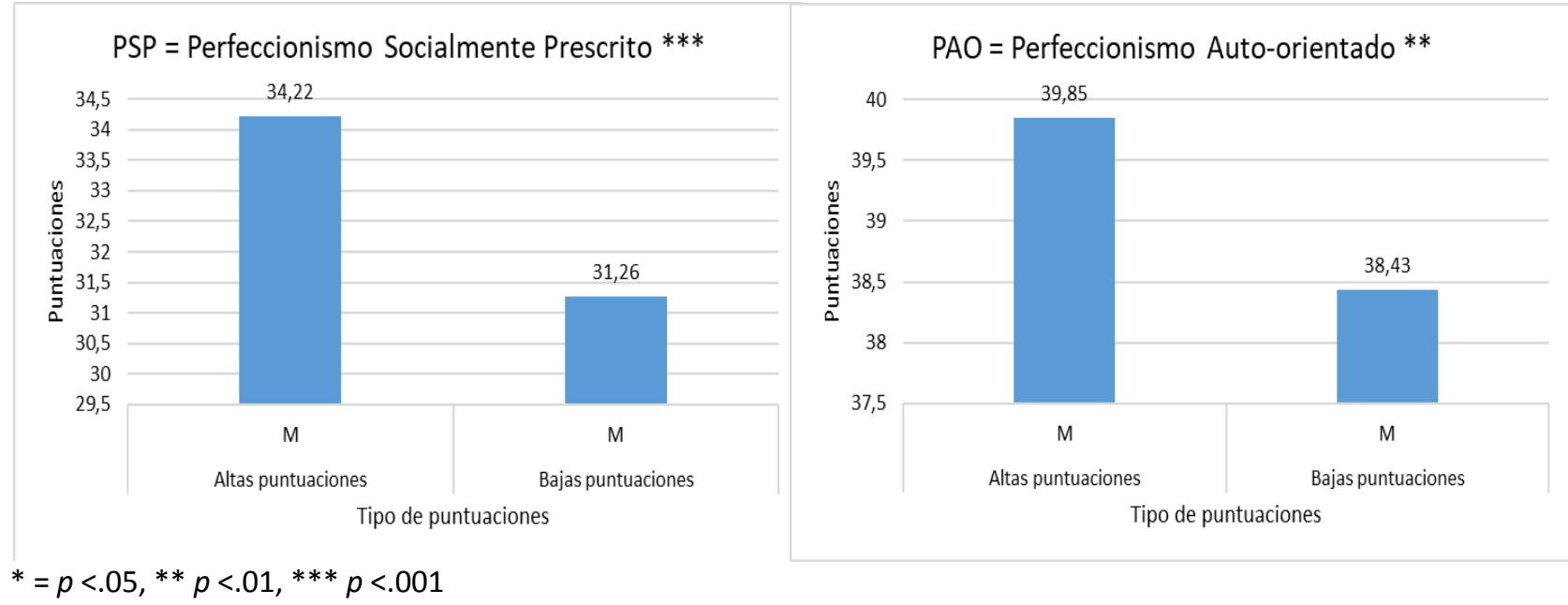

Fuente: Elaboración propia.

\subsection{Regresión logística binaria para la probabilidad de presentar alta puntuación en Ansiedad Conductual en función de las variables de perfeccionismo}

Los modelos resultantes (véase Tabla 6) permiten hacer una estimación correcta del $56.8 \%$ de los casos $(\chi 2=$ 8.98; $p=<.05)$ para la variable PAO y de un $59.5 \%$ de los casos $(\chi 2=37.23 ; p=<.05)$ correspondiente a la variable PSP. El valor de ajuste de los modelos (R2 Nagelkerke) se situó en el .02 para la variable PAO y en .06 para la variable PSP.

Los valores de la odd ratio $(O R)$ fueron superiores a 1 indicando, por tanto, que a medida que aumenta la puntuación en las variables de perfeccionismo aumenta la posibilidad de presentar altas puntuaciones en Ansiedad Conductual, obteniendo valores para la variable PAO de 1.03 y de 1.06 para PSP.

Tabla 6

Regresión logística binaria para la probabilidad de presentar alta puntuación en Ansiedad Conductual en función de las variables de perfeccionismo.

\begin{tabular}{|c|c|c|c|c|c|c|c|c|c|}
\hline Variable & & $x^{2}$ & $\mathrm{R}^{2}$ & B & E.T. & Wald & $p$ & OR & I.C.95\% \\
\hline PAO & $\begin{array}{l}\text { Clasificados } \\
\text { correctamente: } \\
56.8 \%\end{array}$ & 8.98 & .02 & .03 & .01 & 8.83 & .003 & 1.03 & $1.01-1.05$ \\
\hline & Constante & & & -1.07 & .44 & 5.87 & .015 & .34 & \\
\hline PSP & $\begin{array}{l}\text { Clasificados } \\
\text { correctamente: } \\
59.5 \%\end{array}$ & 37.23 & .06 & .06 & .01 & 35.03 & $<.001$ & 1.06 & $1.04-1.09$ \\
\hline & Constante & & & -1.96 & .37 & 27.20 & $<.001$ & .14 & \\
\hline
\end{tabular}

Nota: $\mathrm{PAO}=$ Perfeccionismo Auto-orientado, PSP $=$ Perfeccionismo Socialmente Prescrito, $\chi 2$ = Chi cuadrado; R2 = Cuadrado de Nagelkerke; $\mathrm{B}=$ Coeficiente de regresión; E.T.= Error estándar; Wald = Prueba de Wald; $p=$ Probabilidad; OR = Odd ratio; I.C. = Intervalo de confianza al 95\%.

Fuente: Elaboración propia. 


\subsection{Modelo de regresión logística binaria para la probabilidad de presentar alta puntuación en Ansiedad Conductual en función de las variables de perfeccionismo incluyendo todas las variables}

La Tabla 7 presenta el modelo de regresión logística binaria para Ansiedad Conductual resultante tras incluir las variables de perfeccionismo, en donde los resultados muestran que la proporción de casos clasificados correctamente es del 59.5\% ( $\chi 2=37.23 ; p=<.05)$. Los componentes del modelo expresados por la OR permiten pronosticar una alta ansiedad escolar en base a Ansiedad Conductual, siendo 1.06 veces mayor la probabilidad de manifestar ansiedad escolar por cada punto que se incrementa la puntuación en la variable PSP.

Tabla 7

Modelo de regresión logística binaria para la probabilidad de presentar alta puntuación en Ansiedad Conductual en función de las variables de perfeccionismo incluyendo todas las variables.

\begin{tabular}{|c|c|c|c|c|c|c|c|c|c|}
\hline Variable & & $\chi^{2}$ & $\mathrm{R}^{2}$ & B & E.T. & Wald & $p$ & OR & I.C.95\% \\
\hline PSP & $\begin{array}{l}\text { Clasificados } \\
\text { correctamente: } \\
59.5 \%\end{array}$ & 37.23 & .06 & .06 & .01 & 35.03 & $<.001$ & 1.06 & 1.04-1.09 \\
\hline & Constante & & & -1.96 & .37 & 27.20 & $<.001$ & .14 & \\
\hline
\end{tabular}

Nota: $\mathrm{PSP}=$ Perfeccionismo Socialmente Prescrito, $\chi 2$ = Chi cuadrado; $\mathrm{R} 2$ = Cuadrado de Nagelkerke; $\mathrm{B}=$ Coeficiente de regresión; E.T.= Error estándar; Wald = Prueba de Wald; $p=$ Probabilidad; $O R=$ Odd ratio; I.C. = Intervalo de confianza al $95 \%$.

Fuente: Elaboración propia

\subsection{Diferencias en perfeccionismo en estudiantes con altas y bajas puntuaciones en Ansiedad Psicofisiológica}

La Tabla 8 y Figura 3 ofrecen las medias y diferencias estadísticamente significativas en perfeccionismo halladas en estudiantes con altas y bajas puntuaciones en ansiedad escolar en el Factor IV (Ansiedad Psicofisiológica) del IAES.

Las diferencias halladas resultaron significativas en PSP $=$ Perfeccionismo Socialmente Prescrito, con una magnitud media para esta dimensión $(d=.61)$ y en PAO = Perfeccionismo Auto-orientado, con una magnitud baja $(d=.42)$. El alumnado con altas puntuaciones en Ansiedad Psicofisiológica puntuó significativamente más alto que sus iguales con bajas puntuaciones.

Tabla 8

Diferencias en perfeccionismo en estudiantes con altas y bajas puntuaciones en Ansiedad Psicofisiológica.

\begin{tabular}{|c|c|c|c|c|c|c|c|c|c|c|}
\hline \multirow[t]{2}{*}{ Variable } & \multicolumn{2}{|c|}{$\begin{array}{l}\text { Prueba } \\
\text { Levene }\end{array}$} & \multicolumn{2}{|c|}{$\begin{array}{c}\text { Altas } \\
\text { puntuaciones }\end{array}$} & \multicolumn{2}{|c|}{ Bajas puntuaciones } & \multicolumn{4}{|c|}{$\begin{array}{c}\text { Significación estadística y magnitud } \\
\text { diferencias }\end{array}$} \\
\hline & $F$ & $P$ & $M$ & $D E$ & $M$ & $D E$ & $t$ & g.l. & $p$ & $d$ \\
\hline PSP & 3.76 & .053 & 34.67 & 6.26 & 30.63 & 7.07 & -8.41 & 773 & $<.001$ & .61 \\
\hline PAO & .40 & .527 & 40.62 & 6.37 & 37.91 & 6.47 & -5.87 & 773 & $<.001$ & .42 \\
\hline
\end{tabular}

Nota: PSP = Perfeccionismo Socialmente Prescrito, PAO = Perfeccionismo Auto-Orientado

Fuente: Elaboración propia. 
Figura 3

Diagrama de barras de las diferencias en perfeccionismo en estudiantes altas y bajas puntuaciones en Ansiedad Psicofisiológica,

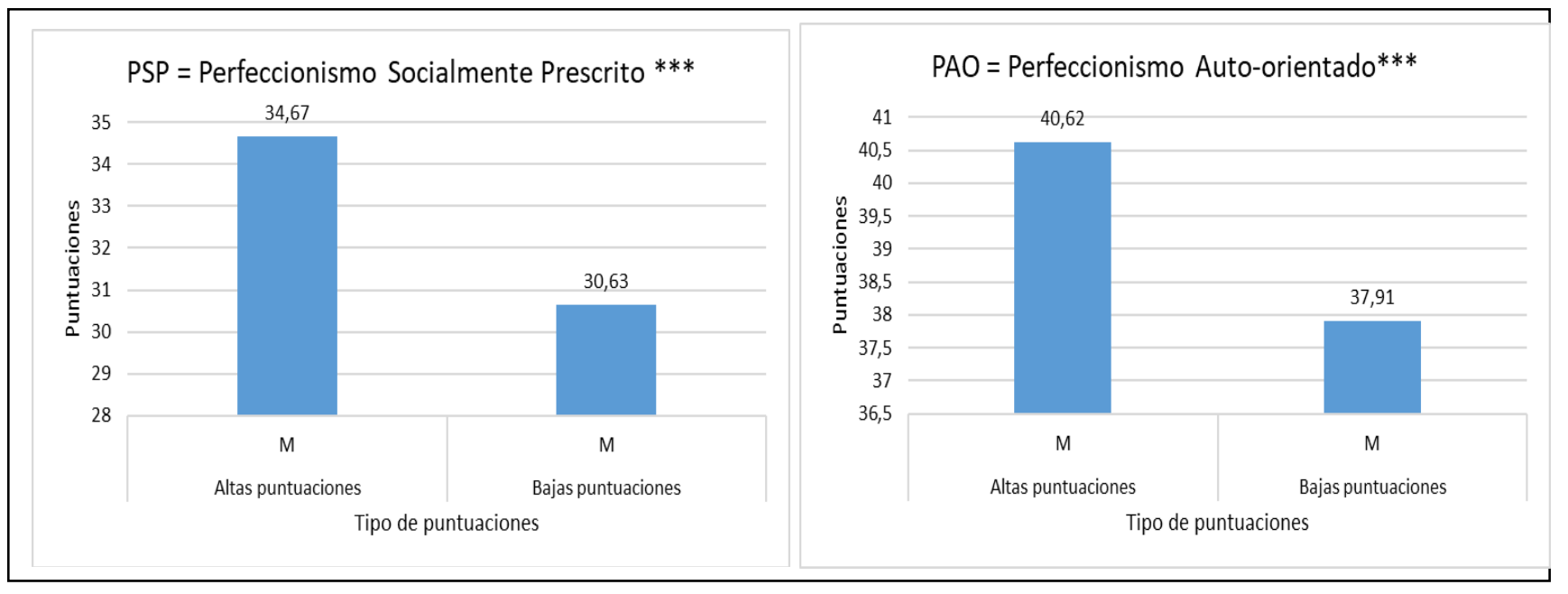

$*=p<.05, * * p<.01, * * * p<.001$

Fuente: Elaboración propia.

\subsection{Regresión logística binaria para la probabilidad de presentar alta puntuación en Ansiedad Psicofisiológica en función de las variables de perfeccionismo}

Los modelos resultantes (véase Tabla 9) permiten hacer una estimación correcta del $59.4 \%$ de los casos ( $\chi 2=$ $33.83 ; p=<.05)$ para la variable PAO y de un $62.6 \%$ de los casos $(\chi 2=67.46 ; p=<.05)$ correspondiente a la variable PSP. El valor de ajuste de los modelos (R2 Nagelkerke) se situó en .06 para la variable PAO y en .11 para la variable PSP.

Los valores de la odd ratio $(O R)$ fueron superiores a 1 indicando, por tanto, que a medida que aumenta la puntuación en las variables de perfeccionismo aumenta la posibilidad de presentar altas puntuaciones en Ansiedad Psicofisiológica, obteniendo valores para la variable PAO de 1.06 y de 1.09 para PSP.

Tabla 9

Regresión logística binaria para la probabilidad de presentar alta puntuación en Ansiedad Psicofisiológica en función de las variables de perfeccionismo.

\begin{tabular}{|c|c|c|c|c|c|c|c|c|c|}
\hline Variable & & $\chi^{2}$ & $\mathrm{R}^{2}$ & B & E.T. & Wald & $p$ & $O R$ & I.C.95\% \\
\hline PAO & $\begin{array}{l}\text { Clasificados } \\
\text { correctamente: } \\
59.4 \%\end{array}$ & 33.83 & 0.6 & .06 & .01 & 31.60 & $<.001$ & 1.06 & $1.04-1.09$ \\
\hline & Constante & & & -2.45 & .46 & 27.52 & $<.001$ & .08 & \\
\hline PSP & $\begin{array}{l}\text { Clasificados } \\
\text { correctamente: } \\
62.6 \%\end{array}$ & 67.46 & .11 & .09 & .01 & 60.09 & $<.001$ & 1.09 & $1.07-1.12$ \\
\hline & Constante & & & -2.80 & .38 & 52.34 & $<.001$ & .06 & \\
\hline
\end{tabular}

Nota: $\mathrm{PAO}=$ Perfeccionismo Auto-Orientado, PSP = Perfeccionismo Socialmente Prescrito, $\chi 2$ = Chi cuadrado; R2 = Cuadrado de Nagelkerke; $\mathrm{B}=$ Coeficiente de regresión; E.T.= Error estándar; Wald = Prueba de Wald; $p=$ Probabilidad; $O R=$ Odd ratio; I.C. = Intervalo de confianza al 95\%.

Fuente: Elaboración propia. 


\subsection{Modelo de regresión logística binaria para la probabilidad de presentar alta puntuación en Ansiedad Psicofisiológica en función de los variables de perfeccionismo incluyendo todas las variables}

La Tabla 10 presenta el modelo de regresión logística binaria para Ansiedad Psicofisiológica resultante tras incluir las variables de perfeccionismo, en donde los resultados muestran que la proporción de casos clasificados correctamente es del 62.6\% ( $\chi 2=67.46 ; p=<.05)$. Los componentes del modelo expresados por la OR permiten pronosticar una alta ansiedad escolar en base a Ansiedad Psicofisiológica, siendo 1.09 veces mayor la probabilidad de manifestar ansiedad escolar por cada punto que se incrementa la puntuación en la variable PSP.

\section{Tabla 10}

Modelo de regresión logística binaria para la probabilidad de presentar alta puntuación en Ansiedad Psicofisiológica en función de los variables de perfeccionismo incluyendo todas las variables.

\begin{tabular}{|c|c|c|c|c|c|c|c|c|c|}
\hline Variable & & $\chi^{2}$ & $\mathrm{R}^{2}$ & $B$ & E.T. & Wald & $p$ & $O R$ & I.C.95\% \\
\hline PSP & $\begin{array}{l}\text { Clasificados } \\
\text { correctamente: } \\
62.6 \%\end{array}$ & 67.46 & .11 & 09 & .01 & 60.09 & $<.001$ & 1.09 & $1.07-1.12$ \\
\hline & Constante & & & -2.80 & .38 & 52.34 & $<.001$ & .06 & \\
\hline
\end{tabular}

Nota: PSP = Perfeccionismo Socialmente Prescrito, $\chi 2$ = Chi cuadrado; R2 = Cuadrado de Nagelkerke; B = Coeficiente de regresión; E.T.= Error estándar; Wald = Prueba de Wald; $p=$ Probabilidad; $O R=$ Odd ratio; I.C. = Intervalo de confianza al $95 \%$.

Fuente: Elaboración propia

\section{Resultados y discusión}

El perfeccionismo se ha identificado como un factor de vulnerabilidad psicológico y existe un amplio consenso en que está relacionado con el rendimiento humano en áreas como el deporte, la música y entornos de ejecución académica (Patston y Osborne, 2015) y como señalan Carrasco, Belloch y Perpiñá (2010).

Wei, Mallinckrodt, Russell y Abraham, (2004) señalan que a mayores niveles de perfeccionismo, mayor consistencia en la relación existente entre ansiedad y depresión. Resulta lógico entonces, pensar que personas perfeccionistas van a permanecer constantemente estresadas, tensionadas, preocupadas, y con un cansancio excesivo o incluso una falta de motivación, ya que, si hablamos de que son exigentes consigo mismas y con los demás, lo llevarán todo a los extremos, tomando como fracaso algún error en las actividades diarias por más insignificante que pueda parecer.

Rice, Kubal y Preusser (2004), empleando una muestra de niños con edades que oscilaban entre los 9 y los 11 años, hallaron que el perfeccionismo afectaba de manera distinta a chicos y a chicas: en los chicos se relacionaba más con los problemas de conducta y en las chicas predecía en mayor medida los problemas internos, como la ansiedad. En este estudio, la autoestima emergió como una forma adaptativa de perfeccionismo, debido a su asociación con el aumento de la estabilidad emocional, demostrando así, que los aspectos adaptativos y desadaptativos del perfeccionismo sí que existen realmente.

En cuanto a las diferencias en perfeccionismo en estudiantes con altas y bajas puntuaciones en Ansiedad Cognitiva, Conductual y Psicofisiológica, las diferencias halladas en estudiantes resultaron coincidentes y significativas en PSP = Perfeccionismo Socialmente Prescrito y en PAO = Perfeccionismo Auto-orientado, con una magnitud baja para ambas dimensiones. De manera que el alumnado con altas puntuaciones en ansiedad puntuó significativamente más alto que sus iguales con bajas puntuaciones. 
Los resultados obtenidos en la investigación permiten confirmar las dos hipótesis de trabajo, ya que los estudiantes con alta ansiedad escolar, en comparación con sus compañeros con baja ansiedad escolar, presenten puntuaciones significativamente más altas en las dimensiones de perfeccionismo y que a medida que aumentan las puntuaciones en perfeccionismo aumente la probabilidad de presentar alta ansiedad escolar. Datos que resultan concordantes con un gran número de estudios que han demostrado el hecho de que el nivel de Perfeccionismo Auto-Orientado en niños, se asociaba con un posible incremento de los niveles de ansiedad (Essau, Leung, Conradt, Cheng y Wong, 2008; Hewitt et al., 2002; Nobel et al., 2012; Stornelli et al., 2009).

En este sentido, si un niño incrementa su nivel de Perfeccionismo Auto-Orientado en relación con las actividades o situaciones escolares, también estaría aumentando sus posibilidades de manifestar alta ansiedad escolar, debido a su propio afán por evitar el fracaso y por cumplir los objetivos académicos que él mismo, consciente o inconscientemente, se haya impuesto. En estos casos, es posible que otras variables de carácter psicoeducativo sean más importantes a la hora de predecir los altos niveles de ansiedad en dichas situaciones escolares. Además, es importante tener en cuenta, que el aumento del Perfeccionismo Auto-Orientado no lleva consigo obligatoriamente un aumento de los niveles de ansiedad escolar. Es posible que muchos niños catalogados como Perfeccionistas no experimenten cambios en su grado de ansiedad escolar, debido a otros factores protectores que impiden la disminución del ajuste emocional.

En cuanto a la regresión logística binaria para la probabilidad de presentar alta puntuación en Ansiedad Cognitiva, Conductual y Psicofisiológica en función de las variables de perfeccionismo, podemos señalar que los valores de la odd ratio $(O R)$ son coincidentes y fueron superiores a 1 indicando, por tanto, que a medida que aumenta la puntuación en las variables de perfeccionismo aumenta la posibilidad de presentar altas puntuaciones en Ansiedad Cognitiva, Conductual y Psicofisiológica, en PAO = Perfeccionismo Auto-Orientado y en PSP = Perfeccionismo Socialmente Prescrito.

\section{Conclusiones}

Los resultados obtenidos en la investigación permiten concluir que los alumnos adolescentes ecuatorianos con alta ansiedad escolar, en comparación con sus compañeros con baja ansiedad escolar, presentan niveles significativamente más altos de perfeccionismo tanto dirigido hacia ellos mismos como dirigido hacia los demás. Además, a medida que aumentan las puntuaciones en perfeccionismo aumenta la probabilidad de presentar alta ansiedad escolar.

\section{Referencias bibliográficas}

Alderete, A.M. (2006). Fundamentos del Análisis de Regresión Logística en la Investigación Psicológica. Evaluar, $6,52-67$.

Carrasco, A., Belloch, A. y Perpiñá, C. (2010). La evaluación del perfeccionismo: utilidad de la Escala Multidimensional de Perfeccionismo en población española. Análisis y Modificación de Conducta, 36(153), 49-65.

Cohen, J. (1988). Statistical power analysis for the behavioral sciences (2nd ed.). Hillsdale, NJ: Lawrence Earlbaum Associates.

Costello, E. J., Egger, H. L. y Angold, A. (2004). Developmental Epidemiology of Anxiety Disorders. En T. H. Ollendick y J. S. March (Eds.), Phobic and anxiety disorders in children and adolescents: A clinician's guide to effective psychosocial and pharmacological interventions (p. 61-91). Oxford University Press. https://doi.org/10.1093/med:psych/9780195135947.003.0003 
Demyttenaere, K., Bruffaerts, R., Posada-Villa, J., Lepine, J. P., Angermeyer, M. C., Bernert, S., ... y Chatterji, S. (2004). Prevalence, severity, and unmet need for treatment of mental disorders in the World Health Organization World Mental Health Surveys. Journal of the American Medical Association, 291(21),25812590. DOI: 10.1001/jama.291.21.2581.

Essau, C. A., Leung, P. W., Conradt, J., Cheng, H. y Wong, T. (2008). Anxiety symptoms in Chinese and German adolescents: their relationship with early learning experiences, perfectionism, and learning motivation. Depression \& Anxiety, 25(9). 801-810. https://doi.org/10.1002/da.20334

Flett, G.L., Hewitt, P.L., Boucher, D.J., Davidson, L.A. y Munro, Y. (2000). The Child-Adolescent Perfectionism Scale: development, validation, and association with adjustment. Manuscrito inédito. York University, Toronto, Ontario, Canadá.

Fujita S, Dreyer HC, Drummond MJ, Glynn EL, Volpi E y Rasmussen BB. (2009). Essential aminoacid and carbohydrate ingestion before resistance exercise does not enhance postexercise muscle protein synthesis. Journal of Applied Physiology, 106(5), 1739-2009.

https://doi.org/10.1152/japplphysiol.90395.2008

García-Fernández, J. M., Inglés, C. J., Martínez-Monteagudo, M. C., Marzo, J. C. y Estévez, E. (2011). Inventario de Ansiedad Escolar: validación en una muestra de estudiantes de Educación Secundaria. Psicothema, 23(2), 301-307.

Gregory, A. M. y Eley, T. C. (2005). Sleep problems, anxiety, and cognitive style in school-aged children. Infant and Child Development, 14, 435-444. https://doi.org/10.1002/icd.409

Harald-Freudenthaler, H., Spinath, B. y Neubauer, A.C. (2008). Predicting school achievement in boys and girls. European Journal of Personality, 22(3), 231-245. https://doi.org/10.1002/per.678

Hewitt, P. L., Caelian, C. F., Flett, G. L., Sherry, S. B., Collins, L. y Flynn, C. A. (2002). Perfectionism in children: Associations with depression, anxiety, and anger. Personality and Individual Differences, 32(6). 1049-1061. https://doi.org/10.1016/S0191-8869(01)00109-X

In-Albon Ty Schneider S. (2007) Psychotherapy of childhood anxiety disorders: A meta-analysis. Psychother Psychosom, 76(1), 15-24. https://doi.org/10.1159/000096361

Kessler, R. C., Berglund, P., Demler, O., Jin, R., Merikangas, K. R. y Walters, E. E. (2005). Lifetime prevalence and age-of-onset distributions of DSM-IV disorders in the National Comorbidity Survey Replication. Arch Gen Psychiatry, 62(6), 593-602. Erratum in: Arch Gen Psychiatry, 62(7), 768. doi:10.1001/archpsyc.62.6.593

King, N. J. y Bernstein, G. A. (2001). School refusal in children and adolescents: A review of the past 10 years. Journal of the American Academy of Child and Adolescent Psychiatry, 40, 197-205.

Mardomingo, M. J. (2005). Modelos neurobiológicos en el trastorno por déficit de atención e hiperactividad. Revista Española de Pediatría: Clínica e Investigación, 61(6), 455-459

Martínez-Monteagudo, M.C., Inglés, C.J., Trianes, M.V. y García-Fernández, J.M. (2011). Perfiles de ansiedad escolar: Diferencias en clima social y violencia entre iguales. Electronic Journal of Research in Educational Psychiatry, 3, 1023-1042. http://dx.doi.org/10.25115/ejrep.v9i25.1467

Mazzone, L., Ducci, F., Scoto, M. C., Passaniti, E., D'Arrigo, V. G. y Vitiello, B. (2007). The role of anxiety symptoms in school performance in a community sample of children and adolescents. BMC Public Health, 7, 347. https://doi.org/10.1186/1471-2458-7-347

McLoone, J., Hudson, J. L. y Rapee, R. M. (2006). Treating anxiety disorders in a school setting. Education and Treatment of Children, 29(2), 219-242. 
Muris, P., Merckelbach, H., de Jong, P. J. y Ollendick, T. H. (2002). The etiology of specific fears and phobias in children: A critique of the non-associative account. Behaviour Research and Therapy, 40(2), 185195. https://doi.org/10.1016/S0005-7967(01)00051-1

Nobel, R., Manassis, K. y Wilansky-Traynor, P. (2012). The role of perfectionism in relation to an intervention to reduce anxious and depressive symptoms in children. Journal of Rational-Emotive \& Cognitive-Behavior Therapy, 30(2), 77-90. https://doi.org/10.1007/s10942-011-0133-5

Ollendick, T. H. y Seligman, L. D. (2005) Anxiety disorders. In C. Gillberg,R. Harrington,\& H.C.Steinhausen (Eds.), A Clinician's handbookof child and adolescent psychiatry, pp. 144-187. Cambridge: Cambridge University Press.

Orgilés, M., Espada, J.P., García Fernández, J.M. y Méndez, F.X. (2009). Relación entre miedos escolares y síntomas de ansiedad por separación infantil. Revista Mexicana de Psicología, 26, 17-25.

Patston, T. y Osborne, M.S. (2015). The developmental features of music performance anxiety and perfectionism in school age music students. Performance Enhancement \& Health, 4(1-2), 42-49. https://doi.org/10.1016/j.peh.2015.09.003

Putwain, D. W. y Daniels, R. A. (2010). Is the relationship between competence beliefs and test anxiety influenced by goal orientation? Learning and Individual Differences, 20(1), 813. https://doi.org/10.1016/j.lindif.2009.10.006

Rice, K. G., Kubal, A. E. y Preusser, K. J. (2004). Perfectionism and Children's Self-Concept: Further Validation of the Adaptive/Maladaptive Perfectionism Scale. Psychology in the Schools, 41(3), 279290. https://doi.org/10.1002/pits.10160

Rosário, P., Mourão, R., Núñez, J. C., González-Pienda, J. A. y Solano, P. (2008). Storytelling as a prometer of Self-Regulated Learning (SRL) throughout schooling. Enn A. Valle, J. C. Núñez, R. G. Cabanach, J. A, González-Pineda y S. Rodriguez (Eds.), Handbook of instructional resources and their applications in the classroom (pp. 107-122). New York: Nova Science.

Stornelli, D., Flett, G.L. y Hewitt, P.L. (2009). Perfectionism, Achievement, and Affect in Children: A Comparison of Students From Gifted, Arts, and Regular Programs. Canadian Journal of School Psychology, 24 (4), 267283. https://doi.org/10.1177/0829573509342392

Vasa, R. A. y Pine, D. S. (2006). Anxiety disorders. In C. A. Essau (Ed.), Child and adolescent psychopathology: Theoretical and clinical implications (p. 78-112). Routledge/Taylor \& Francis Group.

Wei, M., Mallinckrodt, B., Russell, D. W. y Abraham, T. (2004). Maladaptative Perfectionism as a mediator and moderator between adult attachment and depressive mood. Journal of Counseling Psychology, 51(2), 201212.

Wren, D. G. y Benson, J. (2004). Measuring test anxiety in children: Scale development and internal construct validation. Anxiety, Stress \& Coping: An International Journal, 17(3), 227240. https://doi.org/10.1080/10615800412331292606

Esta obra está bajo una Licencia Creative Commons Attribución-NoCommercial 4.0 International

(c) $)$ EY-NC 